\title{
Salmonella typhimurium Mutants of RfaH- Phenotype: Genetics and Antibiotic Sensitivities
}

\author{
By B. A. D. STOCKER, BONNIE M. MALES AND WAKO TAKANO* \\ Department of Medical Microbiology, Stanford University School of Medicine, Stanford, \\ California 94305 , U.S.A.
}

(Received 18 April 1979)

\begin{abstract}
Transductional mapping, with phage ES18 or ES18.h1, showed that several mutations causing the $\mathrm{RfaH}^{-}$phenotype (defective formation of galactose I and also of more distal units of the lipopolysaccharide core) were located between metE and pep $Q$ in the Salmonella typhimurium linkage map; the affected locus is designated $r f a H$. The mutation of one strain of $\mathrm{RfaH}^{-}$phenotype was located elsewhere, at an unidentified $r f a$ locus. Introduction of an $\mathrm{F}^{\prime}$ plasmid containing the metE segment of the Escherichia coli chromosome into several $r f a H$ mutants restored the 'smooth' $\left(\mathrm{Rfa}^{+}\right)$phenotype. Several $r f a H$ mutations, and that of the phenotypically similar $r f a$ mutant, caused increased sensitivity to bacitracin, polymyxin, novobiocin, nafcillin and oxacillin, as expected if the mutations have no effect on the formation of the part of the lipopolysaccharide core proximal to the galactose units.
\end{abstract}

\section{INTRODUCTION}

Salmonella typhimurium mutants of class galE (lacking UDPgalactose epimerase activity) if grown in the absence of exogenous galactose cannot synthesize UDPgalactose and therefore make lipopolysaccharide (LPS) of type Rc, i.e. galactose-deficient LPS core - see Fig. 1 of the following paper (Lindberg \& Hellerqvist, 1980). This LPS defect results in a characteristic pattern of susceptibility to 'rough-specific' phages, including sensitivity to phages C21 and $\mathrm{Br} 2$ (Wilkinson \& Stocker, 1968; Wilkinson et al., 1972). (C21-sensitive mutants of classes $r f a G$ and $g a l U$ differ from galE mutants in that they are resistant to phage $\mathrm{Br} 2$ and make LPS core lacking the glucose I unit, through lack of, respectively, the relevant glucosyltransferase or ability to synthesize UDPglucose.) Two rough mutants which, though able to make UDPgalactose, showed the same phage sensitivities as galE mutants were termed class $r f a H$ (Wilkinson \& Stocker, 1968). Osborn (1968) found that extracts of these two mutants did not catalyse the transfer of labelled galactose from UDPgalactose to C-3 of glucose I of type Rc (galactose-deficient) LPS, and that, correspondingly, their LPS would accept galactose from UDPgalactose when incubated with extracts of $r \mathrm{fa}^{+}$bacteria. It was surmised (Wilkinson \& Stocker, 1968; Osborn, 1968) that a gene affected in these two mutants, termed $r f a H$, specified an enzyme for the galactosyl-transfer reaction which forms the 'main-chain' galactose unit, galactose I, of the LPS core. Three additional mutants with the same phage sensitivity pattern and with similar in vitro galactosyl-transfer properties were assigned to class $r f a H$ by Kuo \& Stocker (1972); they showed that the affected genes of all five $r f a H$ mutants were located elsewhere than the short $c y s E-p y r E$ segment of the linkage map, which includes nearly all other identified $r f a$ genes. In the following paper, Lindberg \& Hellerqvist (1980) describe chemical and serological analyses of the LPS of the original two $r f a H$ mutants; the results indicate that each of the two mutations causes partial,

* Present address: Faculdade de Medicina de Fundação ABC, Santo André, Brazil. 
but not complete, failure to form the galactose I unit, but also causes partial defects in the reactions for formation of several core units distal to galactose I. We here report that the mutated genes of four of the five above-mentioned $r f a H$ mutants are located between metE and pep $Q$ and that the LPS core defect of several of these mutants is corrected by an $\mathrm{F}^{\prime}$ factor bearing the metE-pep $Q$ segment from the Escherichia coli chromosome (cf. Creeger et al., 1979). The affected gene ( $r f a-658)$ of strain SL3648, the fifth of the previously described $r f a H$ mutants, is located elsewhere (and so should no longer be termed $r f a H$ ).

Defects of the deep part of the LPS core of $S$. typhimurium cause increased sensitivity to some antibiotics (Roantree et al., 1977). We here report changes in antibiotic sensitivities caused by $r f a H$ mutations (or by the mutation of a phenotypically similar strain mutated at a locus other than $r f a H$ ) and compare them with changes caused by galE, galU and $r f a G$ mutations.

\section{METHODS}

Bacterial strains and phages. The strains used are listed in Table 1. Of the five mutants previously described as $r f a H$ (Wilkinson \& Stocker, 1968; Osborn, 1968; Kuo \& Stocker, 1972) note that one, strain SL3648, appears from our transduction results (Table 2) to be mutated in a gene different from that affected in the others; its mutant gene is therefore termed $r f a-658$, instead of $r f a H 658$. The phages used to distinguish smooth and different classes of rough mutant were those described by Wilkinson and his colleagues (1972), together with KB1, active on strains lysogenic for P22 (Boro \& Brenchley, 1971) but not on strains lacking $\mathrm{O}$ antigen (B. A. D. Stocker, unpublished observations). The transducing phages used were: ES18, and its derivative ES18. hI which can be grown on LT2 sublines which retain Fels 2 lysogeny (Kuo \& Stocker, 1970); and P22.HT105/1, and an int derivative of it (Schmieger, 1972). Phage P22 wild-type or P22.sie were used to lysogenize strains which were to be recipients in ES18 or ES18.hl transduction (Kuo \& Stocker, 1970).

Phage and genetic methods. Phage pattern, i.e. sensitivity or otherwise to a variety of smooth-specific, rough-specific and other phages, was determined as described by Wilkinson et al. (1972). Hfr and $\mathrm{F}^{\prime}$ crosses were made by standard methods. For transduction we used the 'drop-on-lawn' procedure (Kuo \& Stocker, 1970). Strains to be used as recipients in ES18 transduction were first made lysogenic for P22 or P22. sie (Kuo \& Stocker, 1970). For selection of $m e t E^{+}$transductants we used defined medium with purified agar (Difco Noble Agar), to minimize vitamin B12 contamination. Transductants acquiring met $E::$ Tn10 transposon insertions were selected on nutrient agar with tetracycline $\left(10 \mu \mathrm{g} \mathrm{ml}^{-1}\right)$. Smooth or $\mathrm{RfaH}^{-}$phenotype, and by inference $r f a$ genotype, of purified transductants was determined by the phage pattern test, saline agglutinability or $\mathbf{O}$ agglutinability in a slide test, and by growth in broth, with uniform turbidity or with heavy deposit and little turbidity. For tests on large numbers of transductants we used only phages 9NA (active on smooth strains even if lysogenic for P22), C21 and a mixture of phages Br60 and Ffm (both active on rough strains). For $p m i$ strains (deficient in phosphomannoisomerase and therefore unable to make $\mathrm{O}$ repeat units unless provided with mannose), the LPS phenotype was determined on mannose-supplemented medium.

Antibiotic sensitivity tests. Minimum inhibitory concentrations were determined by the method of Roantree et al. (1977).

\section{RESULTS}

\section{Approximate mapping of rfaH487 by Hfr crosses}

The $\mathrm{rfaH}$ strain SL1060 (or a mannose-fermenting, presumed $\mathrm{pmi}^{+}$, revertant of it) was used as recipient in crosses with several smooth Hfr donor strains, with selection for donor $\mathrm{Met}^{+}$or $\mathrm{Xyl}^{+}$character and against donor auxotrophic character. [The first two $r f a H$ mutants described, SL1060 and SL1033, were both derived, via a pmi mutant, from strain SL1027, which is smooth and requires methionine because it is met $A$ (Wilkinson \& Stocker, 1968). Some time after isolation of the $r f a H$ mutants, strain SL1027 was found to have a leaky, cryptic metE mutation (Kuo \& Stocker, 1970). However, we found the two rfaH mutants, SL1060 and SL1033 (and their $r f a^{+} p m i$ parent, SL1030), to be phenotypically $\mathrm{MetE}^{+}$(Table 1, footnote*). Crosses involving these two mutants were therefore interpreted on the assumption that they were $m e t E^{+}$.] Several $\mathrm{Hfr}$ crosses in which selection was made for donor $\mathrm{Met}^{+}$character yielded recombinants, many of which were $r f a^{+}$, as indicated by their smooth phage sensitivity pattern and $\mathrm{O}$ agglutinability. The presence or absence of 
Table 1. Derivatives of Salmonella typhimurium LT2 used

Strain no.

Description

Source/reference

Parent strains, rfaH mutants and transductional donors

\begin{tabular}{|c|c|}
\hline SL1027* & $\begin{array}{l}\text { metA22 trpD2 H1-b H2-e,n,x 'cured of Fels 2' flaA66 } \\
\text { rpsL120xyl-404 metE551 }\end{array}$ \\
\hline SL1030* & As SL1027 but metE m $^{+}$mi-404 \\
\hline SL1033* & As SL1030 but $r f a H 481$ \\
\hline SL1060* & As SL1030 but $r f a H 487$ \\
\hline SL1741 & As SL1060 but carries $\mathrm{F}^{\prime}$-lac-ataA-P22.HT105/1 \\
\hline $\begin{array}{l}\text { SL3581 } \\
\text { SL3635 } \\
\text { SL3648 }\end{array}$ & $\begin{array}{l}\text { his }(r f b) 519_{\Delta} \operatorname{Str}^{\mathrm{R}} \text { galK462 }\left(\mathrm{F}^{\prime} 8-g a l\right) \\
\text { As SL3581 but } r f a H 655 \\
\text { As SL3581 but } r f a-658\end{array}$ \\
\hline $\begin{array}{l}\text { SL3625 } \\
\text { SL3657 }\end{array}$ & $\begin{array}{l}\text { his C } 527 \text { xyl-416 flaA } 401 \\
\text { As SL } 3625 \text { but } r \text { faH } 659\end{array}$ \\
\hline SL1772 & proB25 $5_{\Delta}$ pepP2 rfaH487 \\
\hline
\end{tabular}

Strains used as transductional recipients

$\begin{array}{ll}\text { metE47 } & \text { metE47 } \\ \text { SL1723 } & \text { metE47(P22) }\end{array}$

TA17 hisR1223 hisD2473 metE338

SL1745 As TA17 but carries P22

TN88 proB25 1 pepQ1 pepP2 metE1355

SL1763 As TN88 but carries P22

SL1766 As TN88 but carries P22.HT105/1

TN87 proB25 $\triangle$ pepQI pepPl

SL1265 As TN87 but carries P22. sie and metE862::Tn10

SL1266 As TN87 but carries P22. sie and metE884::Tn10

Wilkinson et al. (1972)

Wilkinson et al. (1972)

Wilkinson \& Stocker (1968)

Wilkinson \& Stocker (1968)

From SL1060 by $F^{\prime}$ transfer

Kuo \& Stocker (1972)

Kuo \& Stocker (1972)

Kuo \& Stocker (1972)

Vary \& Stocker (1973)

Kuo \& Stocker (1972)

From TN88 by transduction (Table 3, cross 4)

$F^{\prime}$ strains

TR562 hisG200 metE338 ilvC40I pur-847 ( $\left.\mathrm{F}^{\prime} 14\right)$

TR563 As TR562 but hisC117 instead of hisG200

TR564 As TR562 but hisC342 instead of hisG200

McHugh \& Miller (1974)

McHugh \& Miller (1974)

McHugh \& Miller (1974)

Miller \& Roth (1971)

Miller \& Roth (1971)

Miller \& Roth (1971)

* The 'leaky' metE55I mutation of strain SL1027 causes a partial requirement for methionine or vitamin B12, evident when the effect of the metA mutation is reversed by provision of homocystine; its existence was discovered (Kuo \& Stocker, 1970) after the isolation of pmi mutant SL1030 from SL1027. SL1030 and its $r f a H$ mutants SL1033 and SL1060 are phenoty pically MetE ${ }^{+}$, either by 'reversion' of metE551 or because the metE mutation occurred after the isolation of SL1030.

$\dagger$ Strain SL3648, previously regarded as an $r f a H$ mutant because of its phage sensitivity pattern and in vitro galactosyl-transfer character (Kuo \& Stocker, 1972), appears from transduction (Table 2) to be mutated at a separate locus and is therefore now indicated as $\mathrm{rfa}-658$ instead of $\mathrm{raH} 658$.

$r \mathrm{fa}^{+}$recombinants indicated that $\mathrm{rfaH}$ was transferred early by donors of types HfrK3, HfrK6, HfrK13, HfrK14, HfrK19, HfrA, HfrH10 and HfrH4, but not by HfrK10 (data not shown). From the origins of transfer of these donors (Sanderson et al., 1972) we infer that rfaH487 of SL1060 is between about unit 83 and unit 96 on the recalibrated S. typhimurium linkage map (Sanderson \& Hartman, 1978).

\section{Transduction by phage ES1 8 or P22 grown in rfaH mutant SL1060}

Phage ESI8 or ES18.h1 grown on SLI060 ( $r f a H 487)$ was used to evoke prototrophic transductants from strains with auxotrophic mutations mapping in the 83 to 96 unit segment of the linkage map. No $r f a H$ clones were detected amongst transductants derived from $m e t B, m e t F, \arg H, \arg C$, hem $C$ or $i l v$ recipients (data not shown). When the recipient was metE47 or metE338, cotransduction of the rfaH487 allele of SL1060 with met $E^{+}$was observed, usually at frequencies of about $25 \%$ (Table 2, crosses 1,2 and 3 ). To test whether 
Table 2. Cotransduction of rfaH with metE $E^{+}$

\begin{tabular}{|c|c|c|c|c|c|c|}
\hline \multirow[b]{2}{*}{$\begin{array}{c}\text { Cross } \\
\text { no. }\end{array}$} & \multicolumn{2}{|c|}{ Donor } & \multicolumn{2}{|c|}{ Recipient } & \multirow[b]{2}{*}{ Phage } & \multirow{2}{*}{$\begin{array}{l}\text { metE } E^{+} r f a H / \\
\text { total } m e t E^{+} \\
\text {transductants }\end{array}$} \\
\hline & $\begin{array}{c}\text { Strain } \\
\text { no. }\end{array}$ & $\begin{array}{l}r f a \\
\text { allele }\end{array}$ & $\begin{array}{c}\text { Strain } \\
\text { no. }\end{array}$ & $\begin{array}{l}\text { met } E \\
\text { allele }\end{array}$ & & \\
\hline 1 & SL1060 & rfaH487 & metE47 & metE47 & ES18 & $5 / 44=11 \%$ \\
\hline 2 & SL10 60 & rfaH487 & SL1723 & metE47 & ES18 & $12 / 56=21 \%$ \\
\hline $3 a^{*}$ & SL1060 & rfaH487 & SL1745 & metE338 & ES18 & $13 / 49=27 \%$ \\
\hline $3 b^{*}$ & SL1060 & rfaH487 & SL1745 & metE338 & ES18 & $24 / 100=24 \%$ \\
\hline $4 \dagger$ & SL1741 & rfaH487 & TA17 & metE338 & P22.HT105/1 & $2 / 6=33 \%$ \\
\hline 5 & SL1033 & rfaH48I & SL1723 & metE47 & ES18 & $4 / 14=29 \%$ \\
\hline 6 & SL3635 & rfaH655 & SL1723 & metE47 & ES18.h1 & $3 / 12=25 \%$ \\
\hline 7 & SL 3648 & $r f a-658$ & SL1723 & metE47 & ES18.hI & $0 / 27$ \\
\hline 8 & SL3657 & rfaH659 & SL1723 & metE47 & ES18.h1 & $3 / 12=25 \%$ \\
\hline
\end{tabular}

* Cross 3a, 49 metE+ transductants scored for Rfa phenotype after single-colony re-isolation; cross $3 \mathrm{~b}, 100$ additional transductants scored without purification.

$\dagger$ The transducing phage used was from spontaneous induction in $r f a H 487$ strain SL1060 made lysogenic by introduction of an $\mathrm{F}^{\prime}(\mathrm{P} 22 . \mathrm{HT} 105 / 1)$ plasmid (see text).

Table 3. Distribution of unselected donor alleles in transduction of met $E^{+}$or pep $Q^{+}$from rfaH 487 donors to metE pep $Q$ recipients

The recipient in cross 4 was TN88 (proB pepP pepQ metE) and in the other crosses its P22-lysogenic derivative, SL1763.

\begin{tabular}{|c|c|c|c|c|c|c|}
\hline \multirow{2}{*}{\multicolumn{2}{|c|}{$\begin{array}{l}\text { Cross no. Donor } \\
\text { Selection for }{\text { met } E^{+}}^{-}\end{array}$}} & \multirow{2}{*}{ Phage } & \multicolumn{4}{|c|}{ No. of transductants with donor alleles: } \\
\hline & & & \multirow{2}{*}{$\begin{array}{c}m e t E^{+} \\
0\end{array}$} & \multirow{2}{*}{$\begin{array}{c}m e t E^{+} r f a H \\
0\end{array}$} & \multirow{2}{*}{$\begin{array}{c}\text { metE+rfaH pep } Q^{+} \\
45\end{array}$} & \multirow{2}{*}{$\begin{array}{c}m e t E^{+} p e p Q \\
4\end{array}$} \\
\hline 1 & SL1060 & ES18 & & & & \\
\hline 2 & SL1060 & ES18 & 26 & 13 & 11 & 0 \\
\hline 3 & SL1050 & ES18 & 27 & 5 & 15 & 1 \\
\hline 4 & SL1060 & ES18 & 14 & 3 & 2 & 1 \\
\hline 5 & SL1772 & $\mathrm{ES} 18 . h 1$ & 18 & 5 & 3 & 1 \\
\hline \multicolumn{2}{|c|}{ Selection for pep $Q^{+}$} & & pep $Q^{+}$ & $p e p Q^{+} r f a H$ & pep $Q^{+} r f a H ~ m e t E^{+}$ & pep $Q^{+} m e t E^{+}$ \\
\hline 6 & SL1772 & ES18.hl & 1 & 4 & 6 & 0 \\
\hline 7 & SL1772 & ES18.hI & 6 & 10 & 11 & 0 \\
\hline
\end{tabular}

phage $\mathrm{P} 22$, as well as phage ES18, could cotransduce $\mathrm{rfaH}$ with metE we used the supernatant of a broth culture of strain SL1741, which is strain SL1060 ( $r$ faH487) made lysogenic for $\mathrm{P} 22$ by acquisition of an $\mathrm{F}^{\prime}$-lac-ataA with an integrated $\mathrm{P} 22$.HT105/1 prophage (B. A. D. Stocker, unpublished). The filtered supernatant contained only $9 \times 10^{6}$ plaque-forming units $\mathrm{ml}^{-1}$, but its transducing activity allowed detection of cotransduction of $\mathrm{raH} 487$ with metE $^{+}$(Table 2, cross 4).

Cotransduction tests with three other rfaH mutants and with a phenotypically similar rfa mutant

Cotransduction of $r f a H$ with met $E^{+}$by phage ES18 or ES18.hl, at frequencies of about $25 \%$, was also observed for three other $r f a H$ alleles (Table 2, crosses 5, 6 and 8). [Strain SL3635 is an $r f a H$ mutant of a strain with a deletion of the $r f b$ gene cluster, so that one could not tell whether its $r f a H 655$ mutation prevented addition of $\mathrm{O}$ chains. The met $E^{+}$ rfaH655 transductants derived from the smooth recipient SL1723 (metE, P22 lysogenic) were unaffected by smooth-specific phages 9NA and KB1, which shows that $r f a H 655$ in a $r \mathrm{fb}^{+}$strain prevents addition of $\mathrm{O}$ chains.] All of 27 met $E^{+}$transductants evoked by phage ES18.h1 grown on SL3648 ( $r f a-658$ and of $\mathrm{RfaH}^{-}$phenotype) were smooth, like the recipient (Table 2, cross 7). 


\section{Mapping of $r f a H$ in relation to pepQ}

Selection for met $E^{+}$, using SL1060 ( $\left.r f a H 487\right)$ as donor. Gene pepQ, specifying peptidase Q, is located on the 'late' side of metE, and cotransducible with it by phage P22 at frequencies of 8 to $16 \%$ (McHugh \& Miller, 1974). To map $r f a H$ in relation to pep $Q$ we made use of strain TN88 (= LT2 proB pepP pepQ metE), a proline auxotroph which, because of lack of peptidases $\mathrm{P}$ and $\mathrm{Q}$, cannot use leucylproline as a proline source (McHugh \& Miller, 1974). Phage ES18 grown on SL1060 ( $r f a H 487)$ was used to evoke $m e t E^{+}$transductants from P22lysogenic derivatives of TN88; purified transductants were scored for $r f a H$ and pepQ character. In three of four experiments (Table 3, crosses 2, 3 and 4) in which the donor was SL1060, the frequency of cotransduction with met $E^{+}$was 25 to $48 \%$ for $r f a H 487$ and 15 to $33 \%$ for $p e p Q^{+}$, and the frequency of the $m e t E^{+}$class with donor $r f a H$ but without donor pep $Q^{+}$, compared with the rarity of those with donor pep $Q^{+}$but without donor $r f a H$, indicated the order metE-rfaH-pepQ. One experiment (Table 3, cross 1 ) gave an anomalous result; all of 49 transductants selected as met $E^{+}$had the donor pep $Q^{+}$, and 45 of them also had the donor $r f a H 487$.

Selection for pep $Q^{+}$, using a constructed rfaH487 strain as donor. To look for cotransduction of $r f a H$ and met $E^{+}$with pep $Q^{+}$as selected allele we used the same proB pepP pep $Q$ metE recipient as before, and selected transductants able to grow with leucylproline (Cyclo Chemical Division, Tavenol Laboratories, Los Angeles, Calif. 90001, U.S.A.) (McHugh \& Miller, 1974) as proline source. To avoid selecting pro $^{+}$or $p e p P^{+}$transductants we used as donor not SL1060 but strain SL1772, a met $E^{+}$rfaH487 pep $Q^{+}$transductant derived from strain TN88 (Table 3, cross 4). In two experiments the frequency of cotransduction of $r f a H 487$ with pep $Q^{+}$by phage ES $18 . h I$ was about $82 \%$ and that of met $E^{+}$with pep $Q^{+}$was about $45 \%$, and the relative frequencies of the two classes with only one unselected donor marker clearly indicated the order met E-rfaH-pepQ (Table 3, crosses 6 and 7). Transductants with donor $m e t E^{+}$were also selected in this experiment; the frequency of different transductant classes (Table 3, cross 5) was about the same as when the donor was SL1060 (Table 3, crosses 2, 3 and 4).

\section{'Phenotypic curing' of rfaH strains by $\mathrm{F}^{\prime} 14$}

Construction of rfaH metE recipients. The recent report of Creeger and her colleagues (1979) that the LPS defect and lack of in vitro galactosyl-transferring ability of $r f a H$ mutant SL1060 are 'cured' by introduction of a 'Carbon' plasmid containing an unidentified fragment of the $E$. coli chromosome prompted us to test whether an $\mathrm{F}^{\prime}$ plasmid carrying the met $E$ region of the $\mathrm{K} 12$ chromosome would have the same effect. For this we needed met $E$ rfaH strains as recipients. Our transductants of this character had been discarded, and the previously used proB pepP pep $Q$ met $E$ starting strain had regained the ability to grow with leucylproline as protein source. Two strains of constitution proB pepP pepQ (P22.sie) met $E::$ Tn 10 were therefore derived from strain TN87 (Table 1) by lysogenization, followed by transducing in either of two metE: :Tn10 transposon insertions (Kleckner et al., 1977). ES18 or ES18. $h 1$ lysates of $r f a H$ mutants SL1033, SL3635 and SL3657 and of $r f a-658$ mutant SL3648 were applied to the newly constructed recipient strains, SL1265 and SL1266, and selection was made for the ability to grow on defined medium with methionine and leucylproline, i.e. for $p e p Q^{+}$or $p e p P^{+}$or pro $^{+}$transductants. The pep $Q^{+}$transductants thus obtained included one or more which were still $\mathrm{Met}^{-}$but had acquired the $\mathrm{RfaH}^{-}$characters of donors SL1033, SL3635 and SL3657, and were therefore suitable for the test of 'curing' by an $\mathrm{F}^{\prime}$ factor. None of several pep $Q^{+}$transductants (and none of 11 pep $Q^{+} m e t E^{+}$transductants obtained by selection on unsupplemented defined medium) evoked by phage grown on SL3648 ( $r f a-658)$ had its $\mathrm{RfaH}^{-}$phenotype - which is further evidence that $r f a-658$ is not between pep $Q$ and metE. 
Table 4. Antibiotic sensitivities of (i) $\mathrm{RfaH}^{-}$and $\mathrm{Rfa}^{+}$strains and, for comparison, (ii) different classes of core-defective mutant

(i) $\mathrm{RfaH}^{-}$and $\mathrm{Rfa}^{+}$strains

\begin{tabular}{|c|c|c|c|c|c|c|c|}
\hline \multirow[b]{2}{*}{$\begin{array}{c}\text { Strain } \\
\text { no. }\end{array}$} & \multirow[b]{2}{*}{$\begin{array}{c}\text { LFS } \\
\text { genotype: }\end{array}$} & \multicolumn{6}{|c|}{ (Relative) minimum inhibitory concentration* } \\
\hline & & $\begin{array}{c}\text { Erythro- } \\
\text { mycin }\end{array}$ & Bacitracin & Polymyxin & Novobiocin & Nafcillin & Oxacillin \\
\hline SL1030 & $p m i r f a^{+}$ & $(150)$ & $(500)$ & $(0.5)$ & $(200)$ & $(400)$ & $(200)$ \\
\hline SL1060 & pmi rfaH487 & 0.7 & 0.3 & 0.8 & $<0.5$ & 0.4 & 0.5 \\
\hline SL1033 & pmi rfaH481 & 1.0 & $0 \cdot 3$ & 0.6 & $<0.5$ & 0.4 & 0.5 \\
\hline SL3581 & $r f b_{\Delta} r f a^{+}$ & $(50)$ & $(150)$ & $(0 \cdot 3)$ & $(500)$ & $(50)$ & $(<25)$ \\
\hline SL 3635 & $r f b_{\Delta} r f a H 655$ & $<1.0$ & 0.3 & $<0.3$ & $<0.2$ & $<1.0$ & NT \\
\hline SL 3648 & $r f b_{\Delta} r f a-658$ & $<1 \cdot 0$ & 0.7 & $<0.3$ & $<0.2$ & $<1.0$ & NT \\
\hline SL 3625 & $r \mathrm{fa}^{+}$ & $(100)$ & $(500)$ & $(0 \cdot 7)$ & $(500)$ & $(400)$ & $(500)$ \\
\hline SL 3657 & rfaH659 & $1 \cdot 0$ & $0 \cdot 3$ & $0 \cdot 3$ & $<0.2$ & 0.25 & $<0.2$ \\
\hline (five)§ & $r f a^{+}$ & $(150)$ & $(500)$ & $(0.6)$ & $(800)$ & $(200)$ & $(150)$ \\
\hline (four)|| & rfaH487 & 1.0 & 0.4 & 0.7 & $<0.13$ & 0.75 & 0.7 \\
\hline
\end{tabular}

(ii) Core-defective mutant classes

\begin{tabular}{|c|c|c|c|c|c|c|c|}
\hline $\begin{array}{l}\text { Mutant } \\
\text { class }\end{array}$ & $\begin{array}{c}\text { LPS } \\
\text { chemotype }\end{array}$ & $\begin{array}{l}\text { Erythro- } \\
\text { mycin }\end{array}$ & Bacitracin & Polymyxin & Novobiocin & Nafcillin & Oxacillin \\
\hline$r f a L$ & $\mathrm{Ra}$ & $1 \cdot 0$ & $1 \cdot 3$ & 0.9 & $1 \cdot 4$ & $1 \cdot 1$ & $1 \cdot 1$ \\
\hline$r f a J$ & $\mathrm{Rb} 2$ & 1.0 & $1 \cdot 1$ & 0.6 & 1.4 & $1 \cdot 2$ & $1 \cdot 1$ \\
\hline rfa(R-res-2) & Rb3 & 1.0 & 0.5 & $0 \cdot 1$ & 0.05 & 1.0 & 0.9 \\
\hline galE & Rc & 1.0 & 0.4 & $0 \cdot 3$ & 1.8 & 0.8 & 0.7 \\
\hline$r f a G$ & Rd1 & 0.5 & $0 \cdot 3$ & $0 \cdot 1$ & 0.05 & $0 \cdot 1$ & $0 \cdot 2$ \\
\hline galU & Rd1 & $0 \cdot 2$ & 0.1 & $0 \cdot 3$ & $0 \cdot 1,1 \cdot 0$ & $0 \cdot 2$ & $0 \cdot 2$ \\
\hline$r f a F$ & $\mathrm{Rd} 2$ & $0 \cdot 1$ & 0.2 & $0 \cdot 1$ & $<0.05$ & $0 \cdot 2$ & 0.2 \\
\hline$r f a E$ & $\operatorname{Re}$ & 0.03 & $<0.01$ & $0 \cdot 3$ & 0.01 & 0.02 & $<0.05$ \\
\hline
\end{tabular}

* Results in parentheses indicate the minimum inhibitory concentration (m.i.c.) $\left(\mu \mathrm{g} \mathrm{ml}^{-1}\right)$ for $r f a^{+}$(control) strains; other results are the m.i.c. for the indicated $r f a H$ or $r f a-658$ strain relative to the m.i.c. of the corresponding $r f a^{+}$strain $(=1 \cdot 0)$. NT, Not tested.

$\dagger$ Summarized from the data of Roantree $e t$ al. (1977). Results show the mean m.i.c. of $r f a$ strains relative to the m.i.c. of isogenic $r f a^{+}$(control) strains $(=1 \cdot 0)$; where two numbers are shown, these are the extreme values of the same parameter.

¥ See Table 1 for $p m i$ and $h i s(r f b)_{\Delta}$ allele numbers.

$\S$ Strain TN88, its P22-lysogenic derivative SL1763 and three metE ${ }^{+}$but $r f a H^{+}$transductants evoked from SL1763 by ES18 lysate of SL1060.

|| Four metE $^{+}$rfaH487 transductants evoked from SL1763 by ES18 lysate of SL1060.

Phenotype of transconjugants. pep $Q^{+} r f a H$ transductants derived from SL1265 or SL1266 and with the $r f a H$ allele of SL1033, SL3635 or SL3657 were crossed by plate mating with LT2 pur ilv met $E$ his strains carrying $\mathrm{F}^{\prime} 14$ (Table 1); the donor strains had been reisolated on medium selective for $\mathrm{Ilv}^{+} \mathrm{Met}^{+}$character to ensure the presence of the relevant chromosomal segment in the plasmid. Several purified $\mathrm{Met}^{+}$transconjugants for each of the three $r f a H$ alleles were grown up in liquid selective medium (to avoid accumulation of $\mathrm{F}^{-}$segregants) and then used as inoculum for phage pattern tests. All gave smooth phage patterns and were also smooth in their type of growth in liquid medium and in $\mathrm{O}$ agglutinability.

\section{Effect of $\mathrm{RfaH}^{-}$character on antibiotic sensitivities}

To test the effect of $r f a H$ (or $r f a-658$ ) character on antibiotic sensitivities, we determined the minimal inhibitory concentrations (m.i.c.) of six antibiotics for several sets of nearly isogenic strains, each set comprising either $r f a^{+}$parent and $r f a$ mutant(s), or sister $m e t E^{+}$ transductants, $r f a^{+}$like the recipient or with the mutant $r f a H$ gene of the donor, SL1060. 
The mutant alleles tested (Table 4) were the four $r f a H$ shown to becotransducible with $m e t E^{+}$, and $r f a-658$ from the phenotypically similar mutant SL3648. The $r f a$ genes had little or no effect on sensitivity to erythromycin (m.i.c. of $r f a$ strains $\geqslant 0.7$ those of the relevant $r f a^{+}$ control). All the $r f a$ strains showed increased sensitivity to bacitracin (m.i.c. $0 \cdot 3$ to $0 \cdot 7$ of controls), polymyxin (m.i.c. $<0.3$ to 0.8 of controls), novobiocin (m.i.c. $<0.13$ to $<0.5$ of controls), nafcillin (m.i.c. $0 \cdot 25$ to $<1 \cdot 0$ of controls) and oxacillin (m.i.c. $<0 \cdot 2$ to $0 \cdot 7$ of controls).

\section{DISCUSSION}

Two classes of mutant of $\mathrm{RfaH}^{-}$phenotype. The assumption (Wilkinson \& Stocker, 1968; Kuo \& Stocker, 1972) that the five phenotypically similar mutants heretofore called $r f a H$ were affected at a single locus is shown to be incorrect by our cotransduction data (Table 2 and text). The mutations of four strains, found to be cotransducible with metE and pep $Q$ (and clearly between these two loci in the case of the mutation of SL1060; Table 3), may all be in one gene and we propose to retain the symbol $r f a H$ for these mutants. The altered gene, $r f a-658$, of the remaining mutant (SL3648) is not between met $E$ and pep $Q$, since none of $27 m e t E^{+}$, and none of 11 met $E^{+}$pep $Q^{+}$, transductants evoked by phage ES18.h1 grown on SL3648 acquired its $\mathrm{RfaH}^{-}$phenotype (and, as noted above, the $r f a$ mutation of SL3648 is not between cys $E$ and $p y r E$ ). No positive information is yet available as to the location of $r f a-658$ and we consider it premature to assign it a letter symbol.

$\mathrm{RfaH}^{-}$phenotype. We confirmed that the four $\mathrm{rfaH}$ mutants and the rfa-658 mutant SL3648 showed the same pattern of phage sensitivity as galE mutants; but Lindberg \& Hellerqvist (1980) show, in the following paper, that $\mathrm{rfaH}$ strains SL1060 and SL1033 adsorb phage C21 less rapidly than do galE mutants. Our data (Table 4) show that several $r f a H$ mutations and mutation $r f a-658$ have much the same effect on sensitivity to six antibiotics as those recorded (Roantree et al., 1977) as resulting from mutation at $r f a(\mathrm{R}-\mathrm{res}-2)$ or at galE (causing, respectively, failure to form the galactose I unit and failure to form either of the galactose units of the core), as would be expected if the $r f a H$ or $r f a-658$ mutations have no effect on the formation of core units proximal to galactose $I$. The mutations we tested resemble $r f a$ (R-res-2) mutations by causing increased sensitivity to novobiocin, instead of the anomalous decreased sensitivity seen in one galE mutant (Roantree et al., 1977). Osborn (1968) and Kuo \& Stocker (1972) showed that the four $r f a H$ mutants and the phenotypically similar mutant SL3648 are at least partly defective in galactose-transferring activity. All these phenotypic traits would be accounted for if their mutations are, as previously surmised, in the structural gene for the single polypeptide of the transferase which forms the 1,3- $\alpha$-linked galactose I unit of the LPS core (Endo \& Rothfield, 1969).

New evidence as to structure of LPS of mutants of $\mathrm{RfaH}^{-}$phenotype: curing by E. coligenes. In the following paper, Lindberg \& Hellerqvist (1980) describe methylation analysis and serological tests on the LPS of two $r f a H$ mutants. Their results show that in the LPS of these mutants: (i) about $15 \%$ of the core chains lack both the galactose I and the galactose II unit; (ii) many chains which receive a galactose I unit are not continued to form "complete core' chains, but terminate at intermediate points. Thus the function of gene $\mathrm{rfaH}^{+}$is required for the efficient addition not only of the galactose I unit but also of several distal sugars of the core. Gene $r f a H^{+}$may be a positive regulatory gene, or may specify a protein which, though not a component of any glycosyltransferase, yet is required for the efficient action of several of the transferases for addition of core units distal to glucose I. In E. coli $\mathrm{K} 12$ the LPS core unit corresponding in position to galactose I of $S$. typhimurium LPS is a glucose, presumably added by a specific glucosyltransferase, specified by a corresponding gene. However, it was recently shown (Creeger et al., 1979) that a hybrid ColEl plasmid incorporating an unidentified fragment of the $E$. coli $\mathrm{K} 12$ chromosome when transferred to $\mathrm{rfaH}$ mutant SL1060 made it phenotypically $\mathrm{Rfa}^{+}$, as indicated by phage sensitivities and by restoration of in vitro galactosyltransferase activity, presumably because the plasmid 
contains the wild-type form of an $E$. coli gene equivalent to gene $r f a H$ of $S$. typhimurium. Correspondingly, we found that transfer of $E$. coli $\mathrm{F}^{\prime} 14$, carrying the met $E$ region of the $\mathrm{K} 12$ chromosome, to pep $Q^{+}$met $E$ transductant strains with three other $r f a H$ alleles restored the smooth phage sensitivity pattern and $\mathrm{O}$ agglutinability, i.e. $\mathrm{Rfa}^{+}$phenotype. The presence of such a gene in $E$. coli $\mathrm{K} 12$ would be explained if $r f a H$ of $S$. typhimurium is a positive regulatory gene for several $r f a$ loci, or if it specifies a polypeptide needed for the efficient action of several glycosyltransferases.

Function of wild-type allele of gene rfa-658, causing $\mathrm{RfaH}^{-}$phenotype of strain SL3648. As noted above, our transduction results show that the mutated gene $r f a-658$ of strain SL3648 is not located in the metE-pep $Q$ segment. Yet in its phage and antibiotic sensitivities, in its galactosyltransferase activity and ability of its LPS to accept galactose in vitro (Kuo \& Stocker, 1972), this mutant is indistinguishable from the four $r f a H$ mutants. The arguments, above, as to the function of gene $\mathrm{ffaH}^{+}$therefore hold also for the wild-type gene corresponding to $r f a-658$ of strain SL3648, and it seems that mutation at either $r f a H$ or at the locus affected by mutation $r f a-658$ (elsewhere) can interfere with efficient addition of both galactose I and several distal units of the core. Perhaps the protein needed for $\mathrm{RfaH}^{+}$ phenotype is made up of two kinds of polypeptide.

This work was supported by grant no. AI-07168 from the National Institute of Allergy and Infectious Diseases, Department of Health, Education and Welfare. We thank Ashvin Padhya for efficient execution of some transduction experiments, and Charles Miller and John Roth for provision of strains.

\section{REFERENCES}

Boro, H. \& Brenchley, J. E. (1971). A new generalized transducing phage for Salmonella typhimurium LT2. Virology 45, 835-836.

Creeger, E. S., Chen, J. F. \& Rothfield, L. T. (1979). Cloning of genes for bacterial glycosyltransferases. II. Selection of a hybrid plasmid carrying the gene for UDP-galactose:lipopolysaccharide $\alpha .3$ galactosyltransferase. Journal of Biological Chemistry 254, 811-815.

ENDO, A. \& Rothfield, L. T. (1969). Studies of a phospholipid-requiring bacterial enzyme. I. Purification and properties of uridine diphosphate galactose:lipopolysaccharide $\alpha$-3-galactosyl transferase. Biochemistry 8, 3500-3507.

Kleckner, N., Roth, J. \& Botstein, D. (1977). Genetic engineering in vivo using translocatable drug-resistance elements. New methods in bacterial genetics. Journal of Molecular Biology 116, 125-159.

Kuo, T.-T. \& STocker, B. A. D. (1970). ES18, a general transducing phage for smooth and nonsmooth Salmonella typhimurium. Virology 42, 621-632.

Kuo, T.-T. \& Stocker, B. A. D. (1972). Mapping of rfa genes in Salmonella typhimurium by ES18 and P22 transduction and by conjugation. Journal of Bacteriology 112, 48-57.

LindberG, A. A. \& Hellerqvist, C. G. (1980). Rough mutants of Salmonella typhimurium: immunochemical and structural analysis of lipopolysaccharides from $r \mathrm{faH}$ mutants. Journal of General Microbiology 116, 25-32.

McHugh, G. L. \& Miller, C. G. (1974). Isolation and characterization of proline peptidase mutants of Salmonella typhimurium. Journal of Bacteriology 120, 346-371.

Miller, C. G. \& Roth, J. R. (1971). Recessivelethal nonsense suppressors in Salmonella typhimurium. Journal of Molecular Biology 59, 63-75.

OSBORN, M. J. (1968). Biochemical characterization of mutants of Salmonella typhimurium lacking glucosyl or galactosyl transferases. Nature, London 217, 957-960.

Roantree, R. J., Kuo, T.-T. \& MacPhee, D. G. (1977). The effect of defined lipopolysaccharide core defects upon antibiotic resistances of Salmonella typhimurium. Journal of General Microbiology 103, 223-234.

SANDERson, K. E. \& HARTMAN, P. E. (1978). Linkage map of Salmonella typhimurium, Edition V. Microbiological Reviews 42, 471-519.

SANDERson, K. E., Ross, H., ZIEGLER, L. \& MÄKELÄ, P. H. (1972). $F^{+}$, Hfr and $F^{\prime}$ strains of Salmonella typhimurium and Salmonella abony. Bacteriological Reviews 36, 608-637.

SCHMIEGER, H. (1972). Phage P22-mutants with increased or decreased transduction abilities. Molecular and General Genetics 119, 75-88.

VARY, P. S. \& Stocker, B. A. D. (1973). Nonsense motility mutants in Salmonella typhimurium. Genetics 73, 229-245.

Wilkinson, R. G. \& STOCKER, B. A. D. (1968). Genetics and cultural properties of mutants of Salmonella typhimurium lacking glucosyl or galactosyl lipopolysaccharide transferases. Nature, London 217, 955-957.

Wilkinson, R. G., Gemski, P., JR \& StOCKeR, B. A. D. (1972). Non-smooth mutants of Salmonella typhimurium: differentiation by phage sensitivity and genetic mapping. Journal of General Microbiology 70, 527-554. 